\title{
]jfis
}

\section{Design of Autonomous Logistics Transportation Robot System with Fork-Type Lifter}

\section{Jae-Hoon $\mathrm{Cho}^{1}$ and Yong-Tae Kim ${ }^{2}$}

${ }^{1}$ Smart Logistics Technology Institute, Hankyong National University, Anseong, Korea

${ }^{2}$ Department of Electrical, Electronic and Control Engineering, Hankyong National University, Anseong, Korea
Received: Aug. 27, 2017

Revised : Sep. 11, 2017

Accepted: Sep. 12, 2017

Correspondence to: Yong-Tae Kim (ytkim@hknu.ac.kr)

(CThe Korean Institute of Intelligent Systems

(c) This is an Open Access article distributed under the terms of the Creative Commons Attribution Non-Commercial License (http://creativecommons.org/licenses/ by-nc/3.0// which permits unrestricted noncommercial use, distribution, and reproduction in any medium, provided the original work is properly cited.

\begin{abstract}
In this paper, we propose an automated guided vehicle (AGV)-based logistics transportation robot with a fork-type manipulator that are efficient in delivering various and small goods on the shelf. The proposed robot system can be divided into a main server part, a drive control part, and a fork lifter motion control part. The main server part includes the path and task planning algorithm, a communication system for exchanging information, and a database. The drive control part performs navigation control and position recognition through the camera. The fork lifter motion control part contains a control algorithm for transporting the product and basket on the shelf. To evaluate the performance of the proposed transportation robot system, the driving test was performed and a fork lifter working experiment was carried out. The experimental results show that the performance of the proposed robot system is satisfactory and can be applied to the transportation tasks in the distribution centers.
\end{abstract}

Keywords: Logistics transportation robot, Fork-type manipulator, Fork lifter, Position recognition

\section{Introduction}

As the proportion of logistics costs gradually increases, various logistics technologies are being developed to obtain price competitiveness of products through reduction of logistics costs. Especially, due to the successful commercialization of logistics transportation robots such as Amazon's Kiva System, technology development of logistics robots have attracted great interest. Also, the element technology and robot system that can be applied to various logistics fields are being studied. Many companies are adopting various automation and information systems to increase profits and logistics efficiency.

Automated guided vehicle (AGV) is most actively applied to logistics centers and advanced logistics companies such as Amazon have proven the effectiveness of AGV in logistics warehouses. In addition, drone and automatic vehicles are being developed for the automation of the traditional logistics industry. Transportation robot techniques have been developed to perform order processing more quickly and improve work efficiency. A variety of logistics transportation robots have been developed, including position recognition systems, power systems, operating systems, loading and unloading systems, and collision avoidance functions [1-5]. The logistics transportation robot recognizes the exact position of a storage rack on the 
navigation path and the position of a basket on the shelf of the rack. Therefore, a fork-type lifter is needed to elevate up and down, pick up and move out the product basket from the stack on the storage shelf [4, 5]. In order to reduce installation and maintenance costs, autonomous navigation technologies are also being developed using the markers to identify the real-time location of the robot and to drive the planned route [2, 3].

In this paper, we propose an AGV-based logistics robot that combines fork-type manipulators that are efficient in delivering various pallets and baskets. To maximize the efficiency of logistics transportation, an autonomous transportation robot with peaking, traveling and collaborative working capabilities has been studied. At first, a transportation robot with on a slideway is designed and the efficiency is improved by reducing the unnecessary periodic movement between shelves. Fork-type manipulator is designed by employing a linear motor to reduce control complexity.

The transportation task of the proposed robot is to move to the target rack, stretch the fork on the shelf, lift up and draw the basket, move back the robot, and return the target position. For such operations, the position recognition of robot and shelf supports is required. Therefore, the robot reads the QR code information through the camera at the bottom and can recognize its position in real time, and can run autonomously. By using the QR code three points, the direction of travel can be confirmed, and the optimal distance from the shelf for the robot can be realized by using the $\mathrm{QR}$ code attached to oscillation the front of each shelf. A virtual grid map is used for the path planning in the control of multi-object robots, and a path is created so that each robot does not cross the same coordinates at the same time. Also, the optimal path from the current position to the target point is searched and designed to move to the realtime optimal path.

To evaluate the performance of the proposed transportation robot system, evaluation experiments were carried out. The performance of the running test was evaluated by the error between the planned driving path and the actual driving path. The performance of the repeatability work test was evaluated by measuring the frequency with which incorrect work occurred.

\section{Design of Transportation Robot System}

\subsection{Design of Transportation Mobile Robot}

The transportation robot applied to the logistics center needs driving technology, localization technology and task process technology. Driving technology is to safely transport the objects
Table 1. Specification of the transportation robot

\begin{tabular}{lc}
\hline \multicolumn{1}{c}{ Contents } & Values (mm) \\
\hline Width of robot's frame (d) & 600.0 \\
Length of robot's frame (L) & 700.0 \\
Radius of steerable wheel & 24.4 \\
Radius of active wheel (R) & 50.0 \\
Height of lifter's frame & 1596.0 \\
Length of lifter's frame & 300.0 \\
Width of fork-type element & 196.0 \\
Length of fork-type element & 503.5 \\
\hline
\end{tabular}

to a target location. The location recognition is required for driving and collision avoidance in the dynamic environment where several robots are operating. The task processing technique is to deliver products to an operator using a pallet, a shelf, or a rack, and the behavior of the robots varies depending on the tasks. The most important consideration in the design of these robots is the internal environment of the logistics center and the type of operation. Logistics transportation robots that perform diverse and precise tasks increase their development costs accordingly. Therefore, it may be more important to select and develop the task type and functions required by the robot considering efficiency and cost. Therefore, in this paper, we design an autonomous transportation robot system using a fork lifter for the various kinds of logistics processing.

The driving part of the transportation robot was designed considering the dynamic environment in the logistics center. It has two wheels for driving and four auxiliary wheels to optimize the stable movements. The robot is designed to ensure robustness against oscillation and disturbance during transport. The wheels are made of urethane wheels that are resistant to slip or attrition. BLDC motors are adopted as the main driving part and the capacity of the motor is selected as $200 \mathrm{~W}$ (3000 RPM) in consideration of the body weight of the robot and the maximum conveying weight. The battery selects $24 \mathrm{~V}(10 \mathrm{Ah})$ and supplies the main drive motor, control board and sensor power. Table 1 is the specification of logistics transportation robot. Figure 1 shows the design of the drive unit and the prototype of the proposed transportation robot system. The prototype developed is designed to evaluate only the driving and operating accuracy and does not consider structural stability, strength and durability testing.

In the case of uneven floor, the transportation robot deviates from the path or occupies a specific path. These situations can 


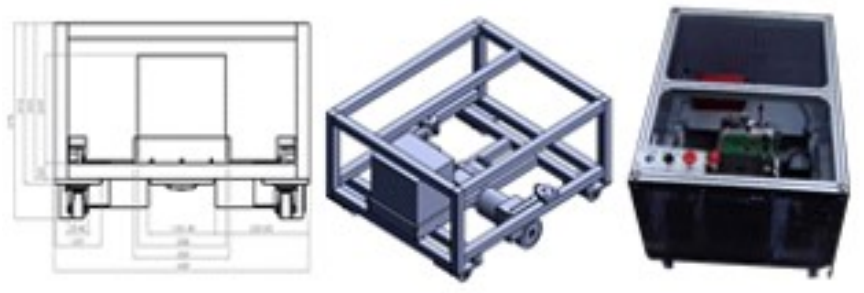

Figure 1. Design and prototype system of the proposed transportation robot.

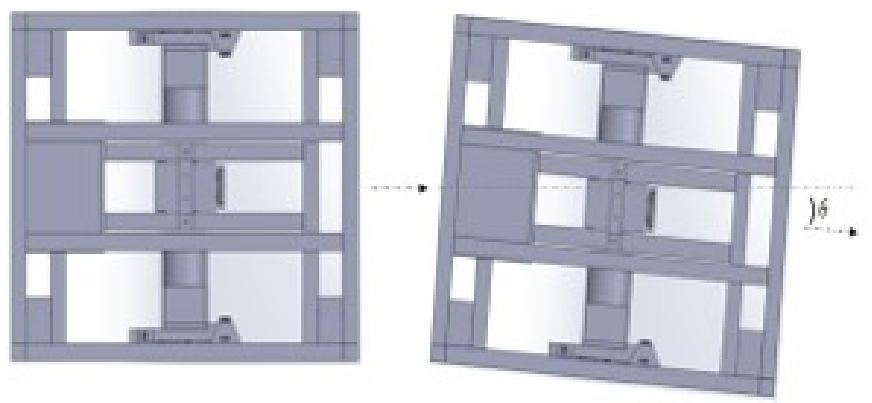

Figure 2. Movement of the transportation robot to the wrong direction.

paralyze the logistics operating system, in which many robots can not operate together. Therefore, a flat and non-slip floor is required for accurate movement and position recognition of the robot. However, the floor construction of the logistics center is very costly and it is difficult to work to overcome any problems on the floor. In the paper, the shock absorber is applied to the wheels so that the wheels can receive the force of the spring in the non-flat region and reach the ground. This shock absorber system prevents the transportation robot from deviating from the planned path.

As shown in Figure 2, the external driving force of the auxiliary wheels and the different actions of both motors cause a problem in the straight movement. Figure 3 shows that when the spring tension of the shock absorber to drive the transportation robot in a non-flat area is so strong, the height of the main wheel becomes higher than the height of the auxiliary wheel. In order to solve this problem, the number of auxiliary wheels is reduced, the design of the wheel position is changed, and the spring tension is adjusted. Also, the analog motor driver was replaced by a digital motor driver.

Relative position and path of robot is calculated using encoder, acceleration and gyro sensor of wheel. Absolute position can be recognized based on image feature points such as landmarks. Amazon's KIVA system has the position recognition method by applying the barcode on the floor of the distribution

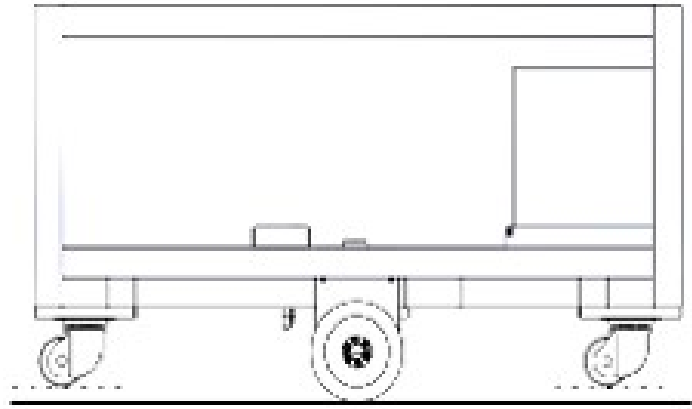

Figure 3. Difference in height between the auxiliary wheel and the main wheel.

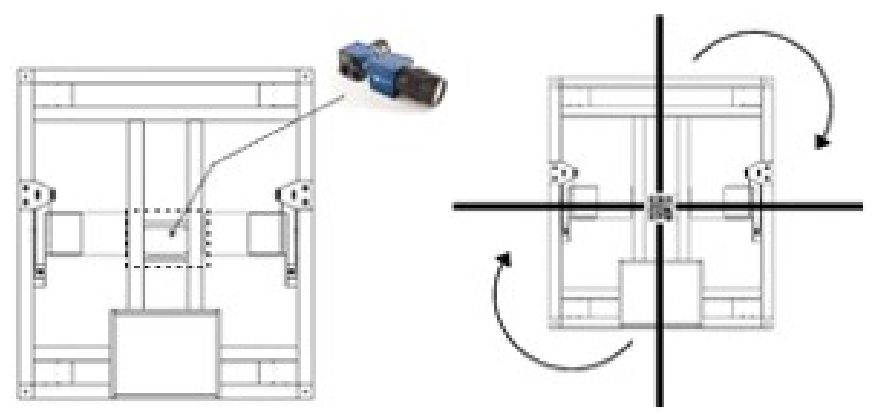

Figure 4. Camera location of the robot for position recognition and driving direction.
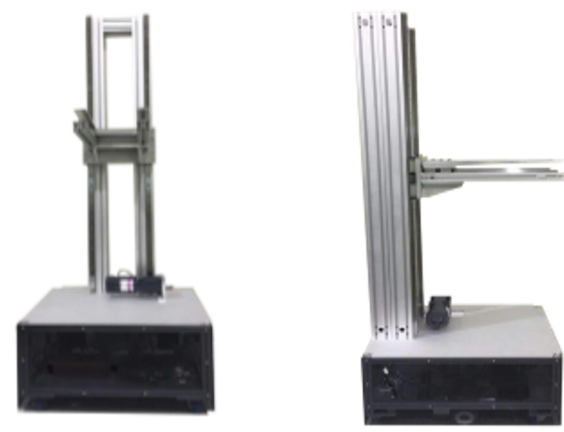

Figure 5. The proposed transportation robot with fork-type lifter.

center. In this paper, the camera is installed at the center of the robot as shown in Figure 4. Based on the image processing, it is possible to travel the path through the detection and tracking of the moving route. The QR code is used as a landmark for position recognition. To calculate the location through $\mathrm{QR}$ code and image processing, we apply Raspberry Pi-3 board and receive path plan using Wi-Fi communication with integrated control server. Figure 5 is a prototype of a transportation robot with the fork lifter. 


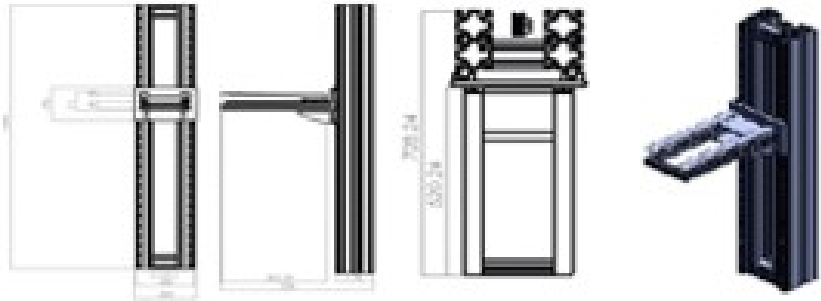

Figure 6. The proposed fork-type lift manipulator.

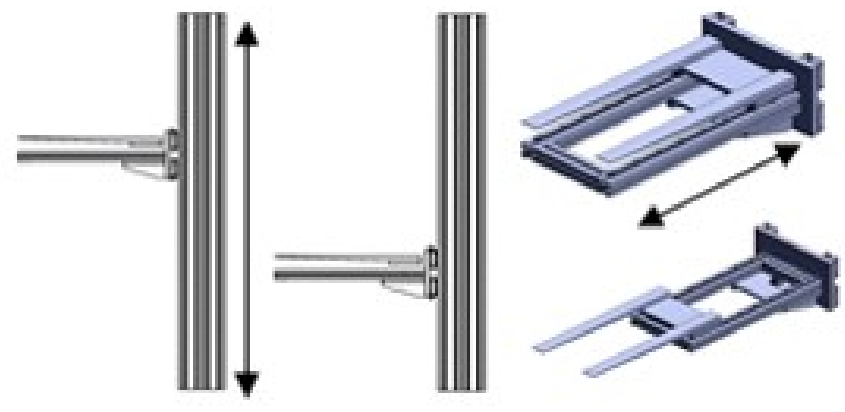

Figure 7. Operating structure for fork and lifter.

\subsection{Design of Fork-Type Lifer}

we design a fork-type lifter that can reduce the sorting time by carrying the shelves different from the pallet or the rack transportation system as shown in Figure 6. Generally, robot arm has flexibility of transporting goods because it has a high degree of freedom, but it is disadvantageous in economical aspect for simple logistics tasks. The fork-type lifter can move up and down, front and rear as shown in Figure 7 and it is possible to lift the shelf with the fork by rising to the height of the target shelf. The shelf is detached from the shelf support when the fork is raised and then retracted. When the operation is performed in the reverse order, the shelf is mounted on the support.

BLDC motors (200 W) are used for lifting up and down movement of the lifter and the stability of the load on the shelf was considered by adopting a chain type. In addition, a proximity sensor is mounted on the base side of the lifter, so that the position of each shelf could be recognized. The operating range of the lifter is choosed similar to the height of a person and designed as $2 \mathrm{~m}$ when it is combined with the logistics transportation robot. Also, its operating capability is designed to carry $1.5 \mathrm{~m}$ of vertical movement, $37 \mathrm{~cm}$ of forward and backward movement, and a maximum of $10 \mathrm{~kg}$ of load.

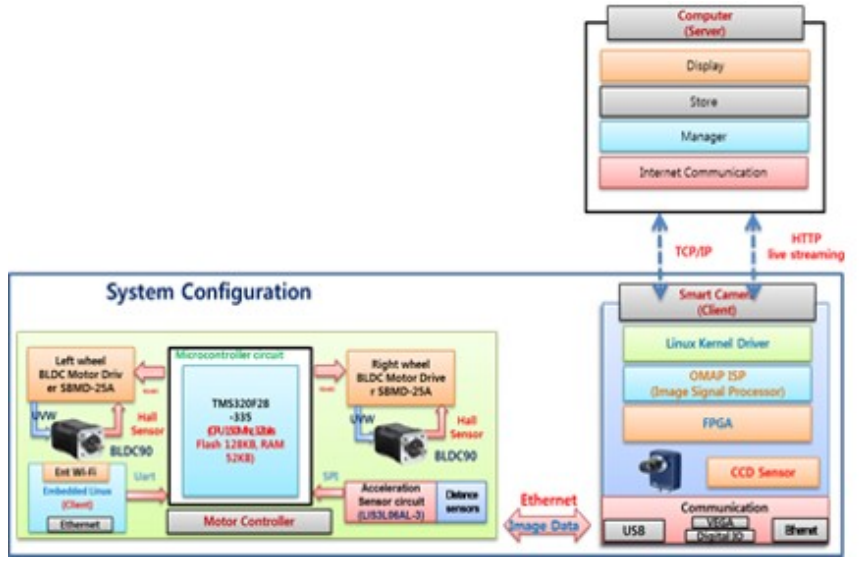

Figure 8. Overall system structure of logistics transportation robot.

\subsection{Design of the Embedded Control System}

The control system of the proposed robot can be divided into a main server, a drive controller, and a forklift motion controller as shown in Figure 8. The main server plans the path of the individual transportation robots and the task schedule of the fork lifter. The informations of paths and schedules are delivered to the robots via Wi-Fi communication. The informations of completed tasks are received and stored in the server's database. Based on the information of the stored database, it continuously calculates the path of the individual transportation robots, and transmits updated path and work plans to the logistics transportation robot in real time.

The drive controller controls the drive motor to travel along the correct path to perform straight run and change of direction. The position information of the $\mathrm{QR}$ code is acquired through the camera at the robot. The current position is calculated through the image processing algorithm of Raspberry Pi-3 and the motor is controlled according to the path error. The forklift motion controller removes the shelf from the specified shelf support using a fork according to the task schedule and then moves it to the specified position. The sensors for the control use a camera for position recognition, a gyro sensor for direction recognition, and an ultrasonic sensor for obstacle detection. Figure 9 shows the software functions of the embedded controller.

The control board shown in Figure 10 was manufactured for control of the transportation robot. The main CPU uses the TMS320F28335 and is connected to the motor driver using relays to prevent reverse voltage. The Bluetooth module for shutdown and passive mode test is connected via UART communication to allow remote control by wireless communication and has IMU sensor for correction of rotation or path tracking 
EMBEDDED H/W CONTROLLER

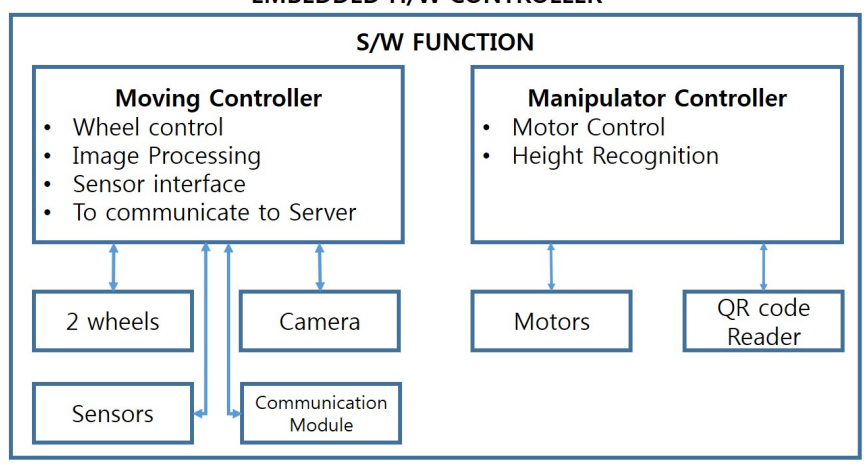

Figure 9. Software functions of the embedded controller.
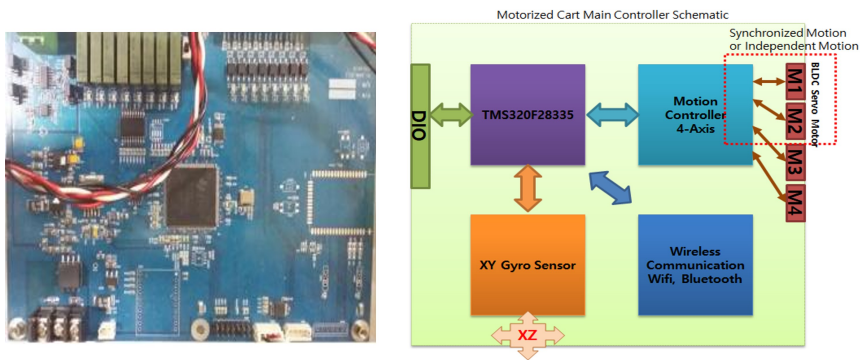

Figure 10. Configuration of the controller board for the transportation robot.

error. Also, it is connected with the acceleration sensor through SPI communication to acquire the attitude information of the robot. The battery uses a $24 \mathrm{~V}$ battery. The BLDC motors are individually controlled through the driver.

\section{Control and Operation System of the Robot}

\subsection{Kinematic Model of the Mobile Robot}

The kinematic model for the driving control of a mobile robot with two wheels is shown in Figure $11[5-\sqrt{6}]$. Assuming that the non-slipping condition is satisfied to simplify the kinematic model of the robot, the velocity equation of the mobile robot is expressed by Eq. (1).

$$
\begin{aligned}
& v=\frac{V_{L}+V_{R}}{2}=\frac{\left(w_{L}+w_{R}\right) r}{2}, \\
& w=\frac{V_{R}-V_{L}}{l}=\frac{\left(w_{R}-w_{L}\right) r}{l},
\end{aligned}
$$

where $l$ is the distance between the centers of two wheels, $r$ is the radius of the wheel, $v$ is the linear velocity of the robot, $w$ is the angular velocity of the robot, $V_{L}$ and $V_{R}$ are the linear velocities of the two wheels, $w_{L}$ and $w_{R}$ are the angular velocities of the two wheels.

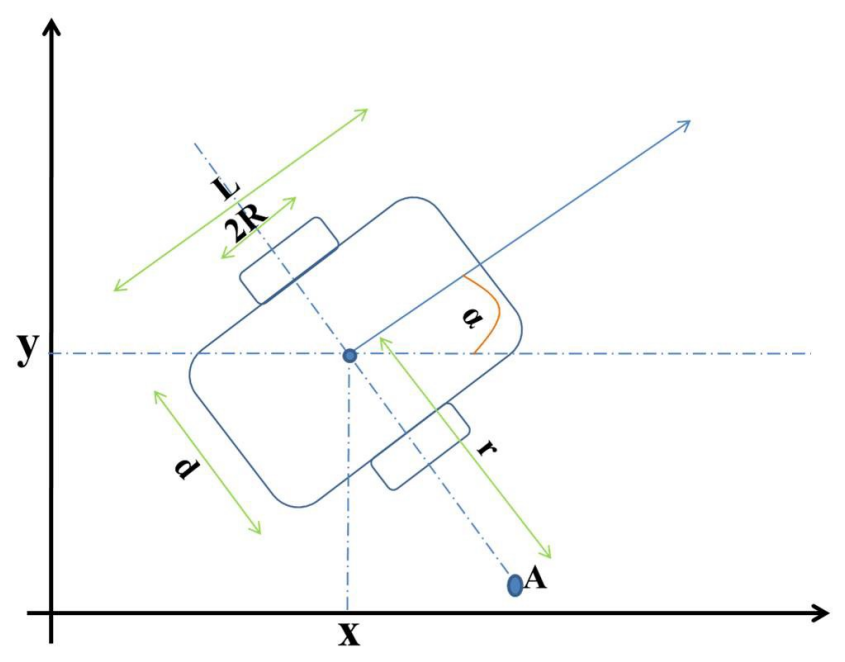

Figure 11. Kinematic model of two-wheeled mobile robot.
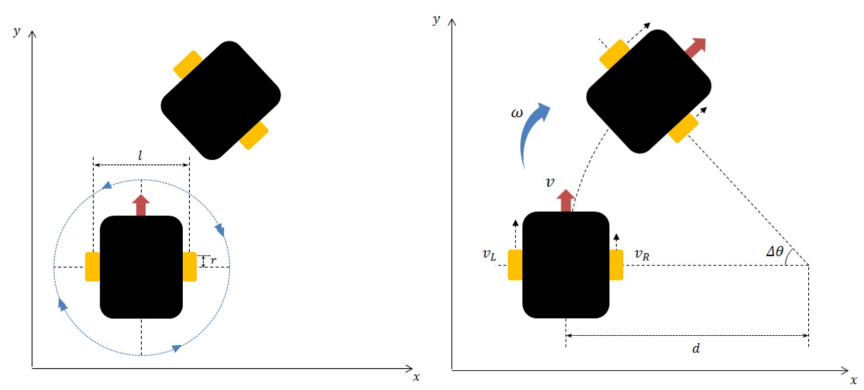

Figure 12. The kinematic model of the transportation robot and the change of the movement trajectory.

Figure 12 shows the kinematic model of the transportation robot and the change of the movement trajectory. $d$ is the radius of the circle generated when the robot moves along the radius of rotation, and $S_{L}$ and $S_{R}$ are the movement distances of each wheel for a given time, then the proportional formula according to the movement trajectory can be obtained as shown in Eq. (2). Here, if the angle of the arc drawn by the robot is $\Delta \theta$ and the moving distance of the center of the robot is $S, \Delta \theta$ and $S$ are shown in Eq. 33.

$$
\begin{aligned}
& S_{R}: d+\frac{l}{2}=S_{L}: d-\frac{l}{2}, \\
& \Delta \theta=\frac{S_{R}-S_{L}}{l}, \quad S=\frac{S_{R}+S_{L}}{2} .
\end{aligned}
$$

\subsection{Control Algorithm for the Robot}

The main server calculates the route, plan tasks and transmits them to the robot through communication. Based on the trans- 


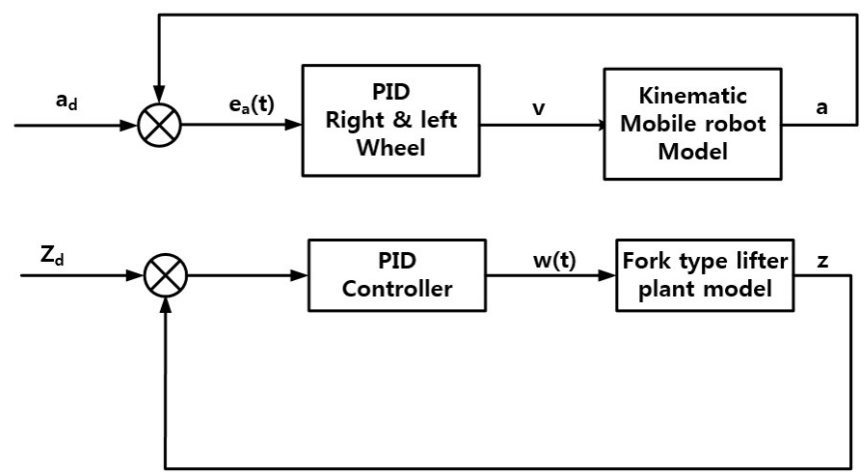

Figure 13. Overall control block diagram for the transportation robot with the fork-type lifter.

mitted path information, the robot starts to travel and transmits the QR code information of the floor surface to the main server in real time. At each $\mathrm{QR}$ code point, a drive controller controls the motor using a PID controller as shown in Figure 13.

When power is applied to the main controller, the register is initialized for operation and the controller performs communication initialization using the GPIO. The mode is set in the RX buffer by SCI communication, and it is driven according to the speed and acceleration value of the user initial setting. These values can be calculated and empirically selected according to the user. If the speed and acceleration values are selected, the yaw value of the gyro sensor is processed and displayed. The controller performs forward and reverse in accordance with the angle change of the yaw value and continuously controls by using the PID control. In addition, the controller switches the direction of left and right based on 90 degrees and performs acceleration and deceleration control for precise direction switching. Figure 14 shows the flowchart of the overall driving mode of the transportation robot. The controller has a separate PID controller according to the mode and the PID gain values were empirically tuned.

\subsection{Task Planning of the Transportation Robot}

The main server plans the path and tasks of the transportation robot. The main server has the location information of the goods including the position of the shelf using the database. Therefore, the main server transmits the optimal driving path and the task sequences of each fork lifter to the transportation robot in order to process the order. The task plan is processed in the fork lifter controller when the robot is completed to reach the target shelf. As shown in Figure 15, the fork lifter controller takes out the basket based on the position information of the

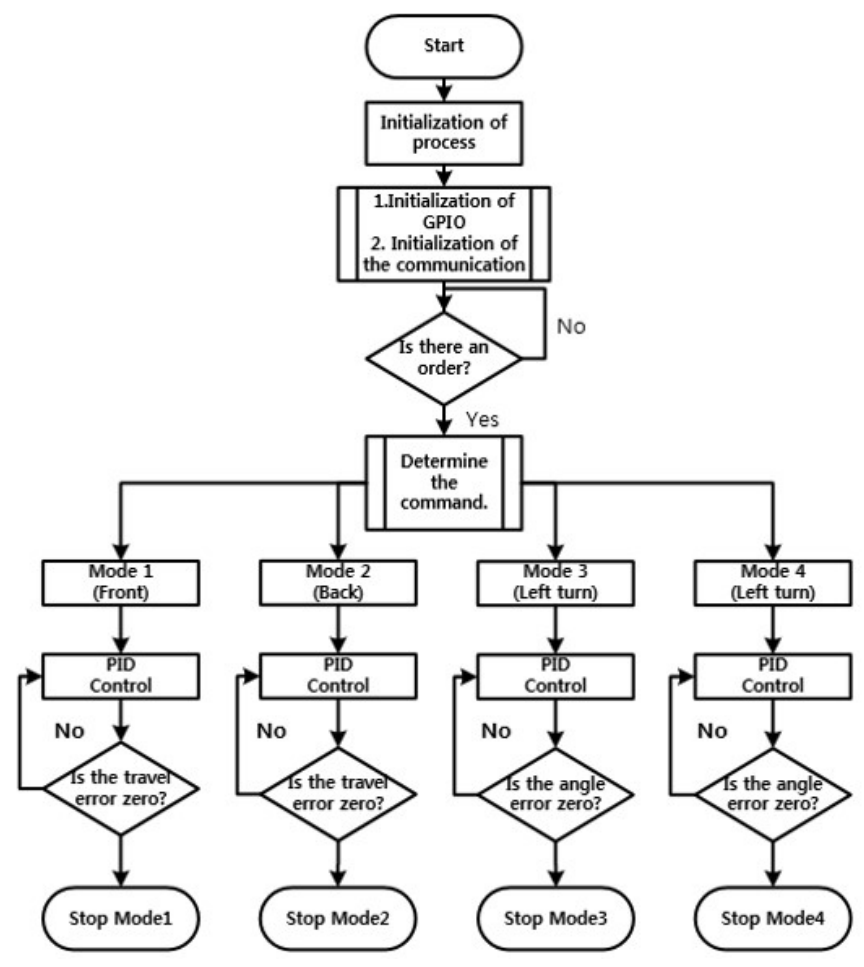

Figure 14. Flowchart of the robot control mode.
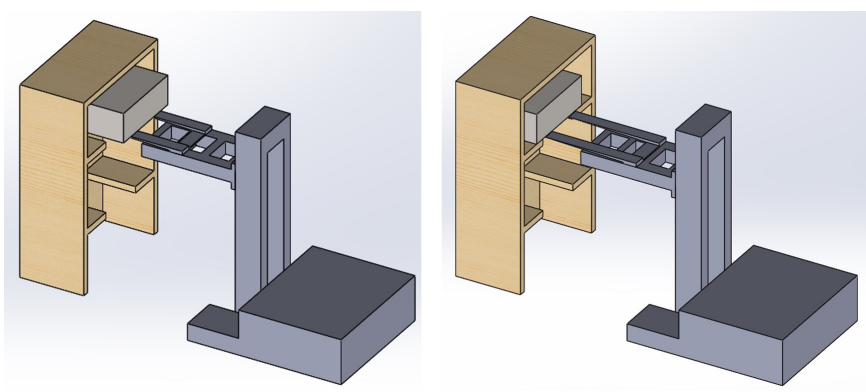

Figure 15. Movement of a basket using a fork lifter of a transportation robot.

shelves and delivers the basket to the operator or returns the basket to the work place. The height of each layer can be known through the sensors installed in each layer.

\subsection{Operating Procedure and Task Management for Mul- tiple Transportation Robots}

The logistics operating system should manage and control a plurality of robots. In addition, the operating system sends and receives data to from the robot to complete each task. Protocol design is needed to reliably transmit and receive command data such as the unique ID of each robot, the current position, the movement path of the robot, the driving speed, the operation mode of the fork lifter, and the task to be performed. The data 
Table 2. The data protocol transmitted from the server to the robot

\begin{tabular}{|c|c|}
\hline byte 0 & $0 x F B$ \\
\hline byte 1 & $0 \times 16$ \\
\hline byte 2 & $0 \times 3 F$ \\
\hline byte 3 & data length $=\mathrm{L}$ \\
\hline byte 4 & $\begin{array}{l}\text { Sign of velocity in manual mode of left } \\
\text { wheel: '十':value }>0,-{ }^{\prime} \text { : value }<0\end{array}$ \\
\hline $\begin{array}{l}\text { byte } 5 \\
\text { byte } 6\end{array}$ & Velocity of left whee \\
\hline byte 7 & $\begin{array}{l}\text { Sign of velocity in manual mode of right } \\
\text { wheel: ' }+ \text { ':value }>0,{ }^{\prime}-' \text { :value }<0\end{array}$ \\
\hline $\begin{array}{l}\text { byte } 8 \\
\text { byte } 9\end{array}$ & Velocity of right wheel \\
\hline byte 10 & Fork-type mode value: in or out \\
\hline byte 11 & Lifter mode value: up or down \\
\hline byte 12 & Correct data confirm \\
\hline byte L & Data of coordinate \\
\hline byte $12+\mathrm{L}$ & $0 x B F$ \\
\hline
\end{tabular}

Table 3. Driving command and operation

\begin{tabular}{cc}
\hline Driving command & Operation \\
\hline F & Forward \\
B & Backward \\
R & Turn right \\
L & Turn left \\
E & Stop \\
U & Lift up \\
D & Lift down \\
O & Fork-type lifter move out \\
I & Fork-type lifter move in \\
\hline
\end{tabular}

protocol transmitted from the main server to the transportation robot is shown in the Table 2. The driving command and the corresponding operations are configured as shown in the Table 3 .

In addition, we propose an operation algorithm for an efficient ordering task of a distribution center. First, the operator scans the bar code of any order history. The information of the scanned order is registered in the main control server. Then, the logistics operating system analyzes the registered order history and identifies the location of the individual items and the information of the transportation robot. The system uses the confirmed information to plan the path and task of the robot

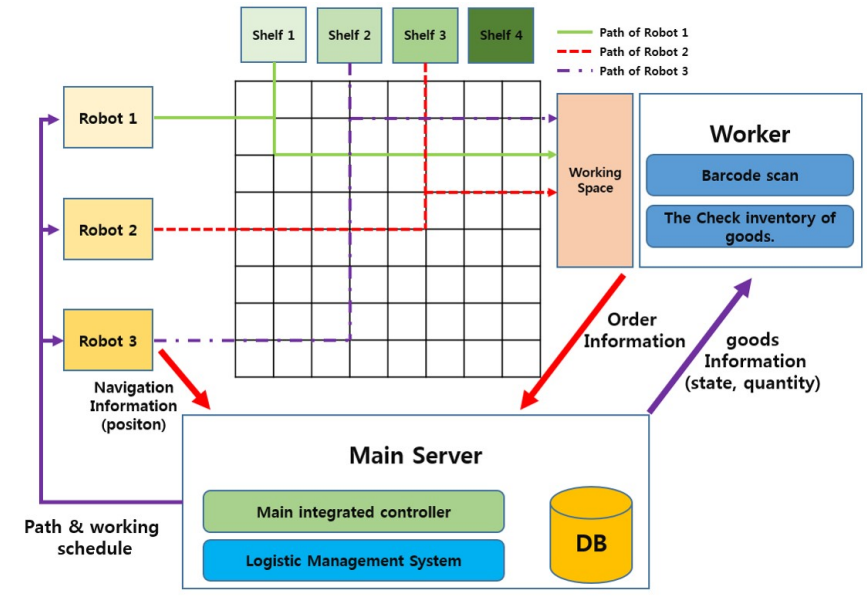

Figure 16. The operation procedure and data information in the logistics center.

and send it to the robot. The robot that receives the planned path and the task moves to the selected basket following the planned path. After picking the basket, the robot delivers it to the operator. The worker pulls out the necessary goods from the robot's basket, takes the barcode, and packs it according to the consumer's order. At this time, the barcode information of the product is transmitted to the operation algorithm and used for the warehouse management and inventory management of the distribution center. Figure 16 shows the operation procedure and data information in the logistics center. Using this information, the operator can be informed about the absence of a specific product, and the manager of the logistics center can easily find out information about which product needs to be supplied to the center.

\section{The Experimental Results}

To evaluate the performance of the developed transportation robot, the driving test was performed 20 times. A QR code and a tracking line are installed on the floor to test the driving performance and an image of the planned route is captured through the camera. The driving error is calculated using the image and image processing algorithm in the Raspberry-Pi. Figure 17 shows the line and QR code installed on the floor and Figure 18 shows the realtime image during driving of the robot.

The driving experiment was carried out with a straight movement between two QR codes and the driving error and angle error were collected at 145 points while driving test. Figure 19 shows the mean and standard deviation calculated through the planned exact path at each point of driving experiment. The 


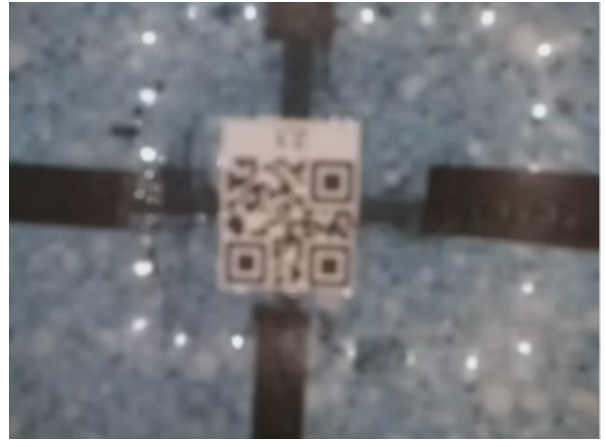

Figure 17. The line and QR code installed on the floor.

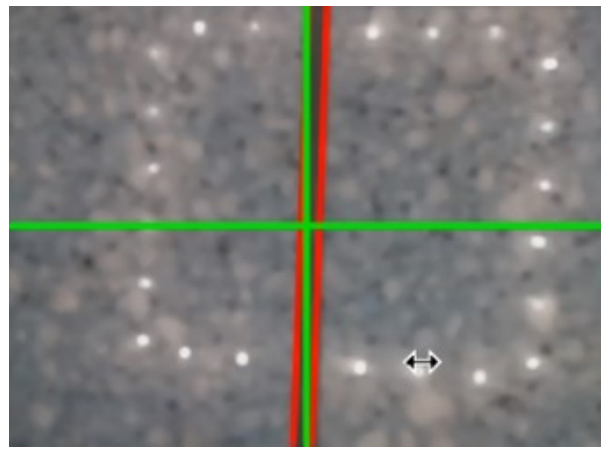

Figure 18. The realtime image during driving of the robot.

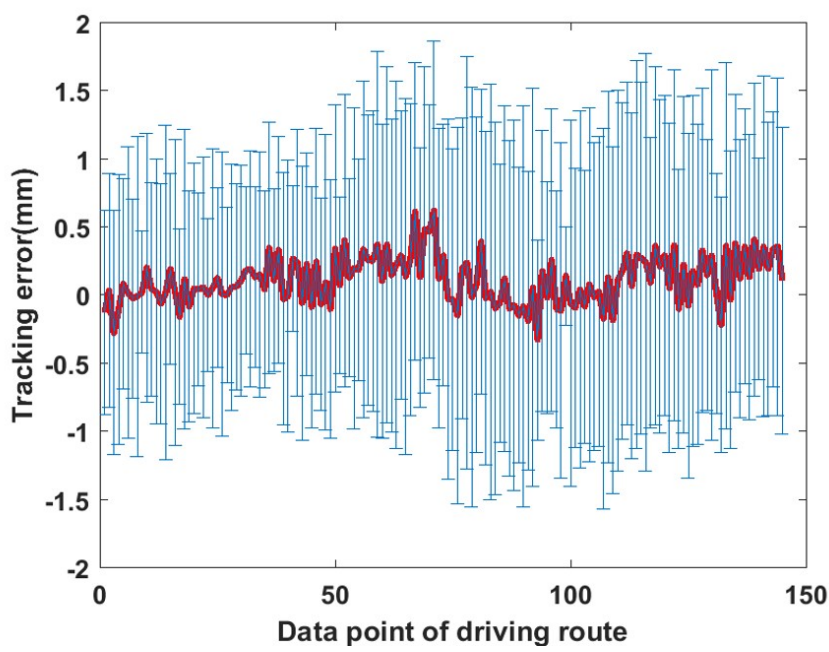

Figure 19. The mean and standard deviation from the planned path in the driving experiment.

experimental results show that the maximum and minimum standard deviations are $1.54 \mathrm{~mm}$ and $0.36 \mathrm{~mm}$, respectively. Figure 20 shows the angle error at each point of driving experiment. Figures 19 and 20 show the good driving performances.

In order to evaluate the performance of the basket transporting operation, fork lifting and loading experiments were carried out 100 times. We show that the developed robot per-

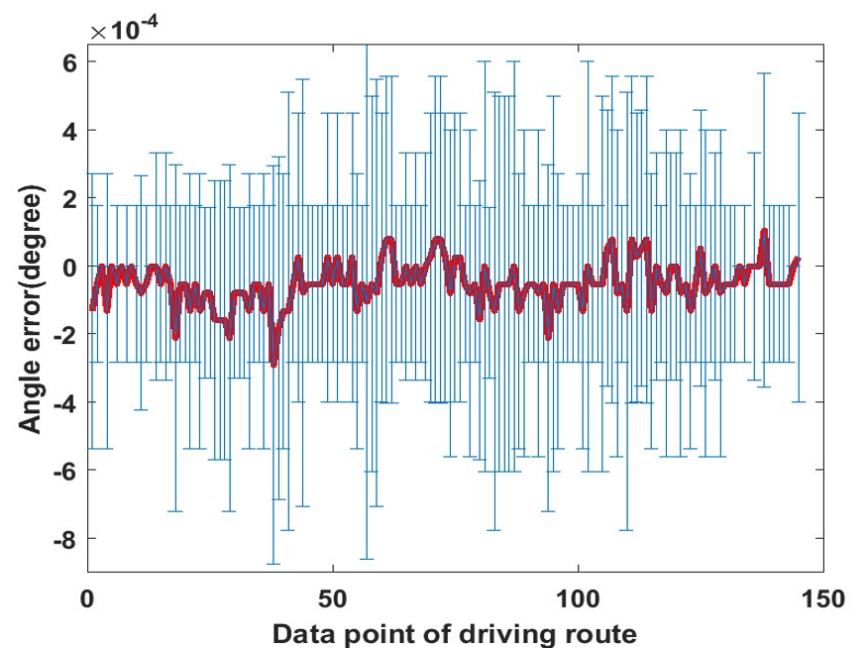

Figure 20. The angle error from the planned path in the driving experiment.

forms the correct operation repeatedly without failure. Figure 21 shows the operation experiment using the fork lifter. Many operation experiments were carried out and showed the desired performance. Therefore, it is expected that the developed transportation robot with fork lifter can be applied to the distribution center to improve the accuracy and efficiency of the logistics transportation operation.

\section{Conclusion}

In this paper, an efficient logistics transportation robot system with fork lifter is proposed. The proposed system is designed including a mobile robot, a main server, and a fork-type lifter. The mobile robot is designed in consideration of stable and efficient movement, and the fork lifter is designed to pull out the basket on the shelf. The main server calculates the overall path and the task plan of the robots and instructs the operation by communicating with each robot. To evaluate the performance of the proposed transportation robot, the driving test was performed and a fork lifter working experiment was carried out. The experimental results show that the performance of the proposed transportation system is satisfactory.

\section{Acknowledgements}

This work was supported by the National Research Foundation of Korea (NRF) grant funded by the Korea Ministry of Education (No. 2015R1D1A1A01060400). 

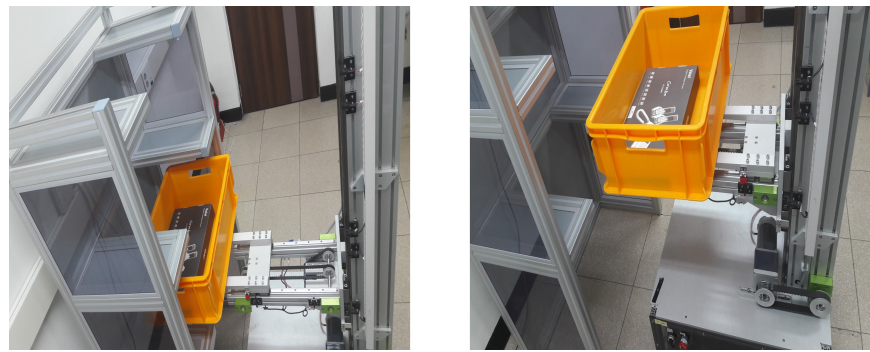

(a)
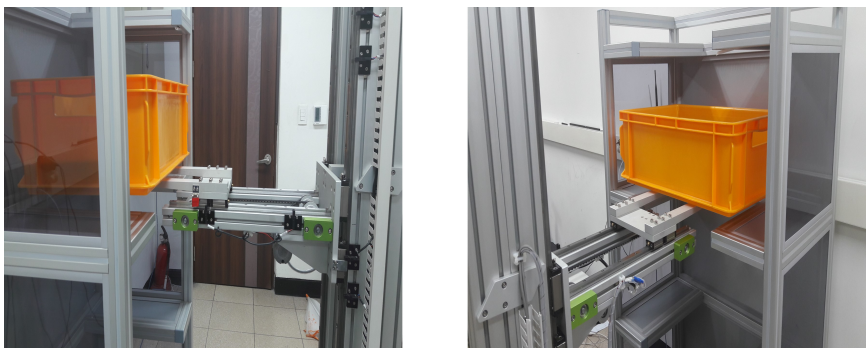

(b)
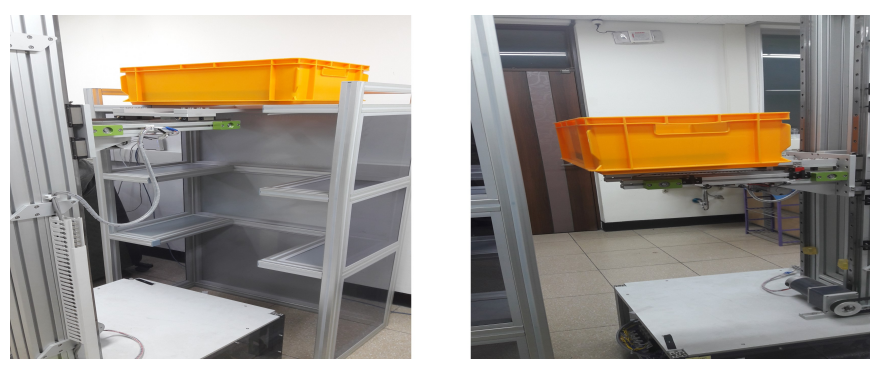

(c)

Figure 21. Operation experiments of the fork lifter. (a) First floor, (b) second floor, and (c) third floor.

\section{References}

[1] Y. M. Kim and Y. T. Kim, "Design of logistics transportation robot based on modular conveyor rack and path planning in logistic center," Journal of Institute of Control, Robotics and Systems, vol. 22, no. 2, pp. 83-88, 2016.

[2] D. H. Jeong, J. I. Park, and Y. T. Kim, "Study on design of mobile robot for autonomous freight transportation," Journal of Korean Institute of Intelligent System, vol. 23, no. 3, pp. 202-207, 2013.

[3] N. T. Truc, E. H. Sun, Y. M. Kim, and Y. T. Kim, "Navigation method using fuzzy line tracking for the transportation robot," in Proceedings of 15th International Symposium on Soft Computing and Intelligent Systems (SCIS) and 2014 Joint 7th International Conference on and Advanced Intelligent Systems (ISIS), Kitakyushu, Japan, 2014, pp. 819-822.

[4] N. V. Vui and Y. T. Kim, "Controller design for the lifting system of transportation robot," Proceedings of KIIS Spring Conference, vol. 26, no. 1, pp. 77-78, 2016.

[5] N. V. Vui, T. H. Luat, and Y. T. Kim, "PID and adaptive controllers for a transportation mobile robot with forktype lifter," International Journal of Fuzzy Logic and Intelligent Systems, vol. 16, no. 3, pp. 216-223, 2016. http://doi.org/10.5391/IJFIS.2016.16.3.216

[6] P. F. Muir and C. P. Neuman, "Kinematic modeling of wheeled mobile robots," Journal of Robotics System, vol. 4, no. 2, pp. 261-340, 1987. http://doi.org/10.1002/rob. 4620040209

[7] A. Bara and S. Dale, "Dynamic modeling and stabilization of wheeled mobile robot," in Proceedings of the 5th WSEAS International Conference on Dynamical Systems and Control, La Laguna, Spain, 2009, pp. 87-92.

[8] F. A. Salem, "Dynamic and kinematic models and control for differential drive mobile robots," International Journal of Current Engineering and Technology, vol. 3, no. 2, pp. 253-263, 2013.

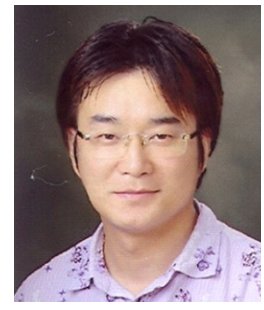

Jae-Hoon Cho received his B.S. and M.S. degrees in Control and Instrumentation Engineering from Hanbat National University, Daejeon, Korea, and Ph.D. degree in Control and Instrumentation Engineering from Chungbuk National University, Cheongju, Korea, in 2002, 2005, and 2011, respectively. He has joined in 2011 as a research professor of Smart Logistics Technology Research Center. His current research interests include intelligent robots, logistics automation, renewable energy and its applications.

E-mail: jhcho@hknu.ac.kr

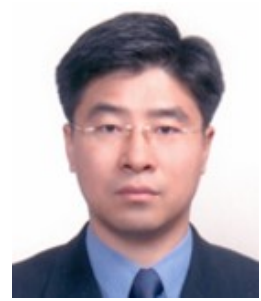

Yong-Tae Kim received his B.S. degree in Electronic Engineering from Yonsei University, Seoul, Korea, and his M.S. and Ph.D. degrees in Electrical and Electronic Engineering from KAIST, Daejeon, Korea, in 1991, 1993, and 1998, respectively. From 1998 to 2000, he was a senior engineering in 
Samsung. In 2006, he was a visiting scholar at Department of Electrical Engineering in University of Illinois at UrbanaChampaign. Since 2002, he has joined the faculties of the Department of Electrical, Electronic and Control Engineering at Hankyong National University, where he is currently a professor. His current research interests include intelligent robots, logistics automation, intelligent control and its applications.

E-mail: ytkim@hknu.ac.kr 